Fritz M. Koller

Programmplaner Physiotherapie

fritz.koller@thieme.de

\title{
Klicken und Schnuppern
}

_ Die Halbwertszeit für Wissen in der Medizin liegt bei circa 5 Jahren. Das heißt: Man müsste die Hälfte von allem, was man vor 5 Jahren gelernt hat, aktualisieren. Damit ist medizinisches Fachwissen Spitzenreiter. Bei Hochschulwissen liegt die Rate allgemein bei circa 5-10 Jahren, bei Schulwissen bei circa 20 Jahren.

_ Um aktuell zu bleiben, besucht man als Physiotherapeut Fortbildung über Fortbildung. Doch woher weiß man, ob alles aktuell ist, was man hinter verschlossener Tür im Fortbildungszentrum erzählt bekommt? Es ist nicht ganz leicht, sicher zu erkennen, ob eine gebuchte Fortbildung aktuell ist. Mehr Transparenz wäre hier wünschenswert. Ein Schnuppern in die Fortbildung würde helfen. Empfeh-

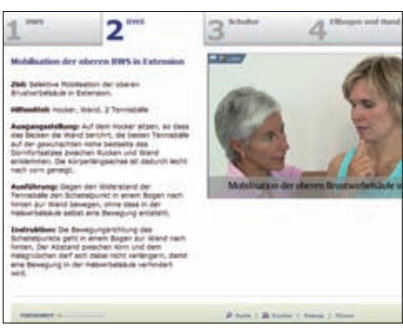
lungen von Kolleginnen und Kollegen ebenso.

_ Das Internet bietet neue Möglichkeiten, in Kurse hineinzuschnuppern. So stellen einige Fortbildungszentren Videos online zur Verfügung. Modern wird es, wenn sogar E-Learning-Kurse zum Schnuppern zusätzlich zur Verfügung stehen. Doch davon gibt es momentan noch wenige.

_ Das ändern wir gerade: Denn wer in E-Learning-Kurse hineinschnuppern möchte, kann dies zum Beispiel auf „physiofortbildung“, dem neuen E-LearningPortal von Thieme unter www.thieme.de/physiofortbildung. Wir freuen uns, wenn Sie vorbeiklicken. Nach 3-jähriger Entwicklungszeit ist physiofortbildung online. Wir haben in den letzten Jahren sehr genau hingehört, was unsere Kolleginnen und Kollegen in den Praxen und Kliniken möchten. Wenn Ihnen physiofortbildung hilft, am Ball zu bleiben, haben wir unsere Arbeit richtig gemacht.

_ Fortbildungen sind das eine, Kongresse ein zweiter Weg, um am Ball zu bleiben. Ich erlebe selten so viele hochmotivierte Physiotherapeuten zur gleichen Zeit am gleichen Ort wie auf einem Kongress. Das nächste Treffen wird der physiokongress sein - vom 27.-29. Januar 2012 in Stuttgart. Merken Sie sich den Termin vor. Es lohnt sich.

Herzlichst, Ihr
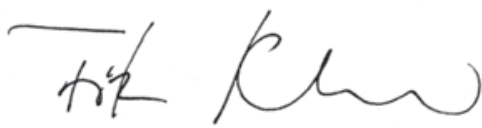

physiofortbildung:

neues E-Learning-Portal

für Physiotherapeuten

\section{ZU GEWVIN NEN}

In jeder physiopraxis werden attraktive Gewinne verlost. Möchten Sie einen ergattern, klicken Sie unter www.thieme.de/physioonline auf „physiopraxis-exklusiv“.

Und das gibt es in dieser Ausgabe zu gewinnen:

Kursplatz

2 Kursplätze „21-Tage-E-MailMotivationstraining“ Seite 47

Bücher

2-mal „Klinische Muster in der Manuellen Therapie“ Seite 28

2-mal „Manuelle Therapie“ Seite 39

3-mal „Arzneimittel pocket 2012“"

Seite 44

3-mal „Schwindel“

Seite 63

und außerdem

3-mal 3 Jonglierbälle

Seite 36

2 Luxus-Kniegelenkmodelle Seite 56

im Refresher

2-mal „Patientenedukation“ 